\title{
Attention-based Extraction of Structured Information from Street View Imagery
}

\author{
Zbigniew Wojna* Alex Gorban $^{\dagger} \quad$ Dar-Shyang Lee $^{\dagger}$ Kevin Murphy $^{\dagger}$ \\ Qian $\mathrm{Yu}^{\dagger}$ Yeqing $\mathrm{Li}^{\dagger}$ Julian $\mathrm{Ibarz}^{\dagger}$ \\ * University College London \\ $\dagger$ Google Inc.
}

\begin{abstract}
We present a neural network model - based on Convolutional Neural Networks, Recurrent Neural Networks and a novel attention mechanism - which achieves $84.2 \%$ accuracy on the challenging French Street Name Signs (FSNS) dataset, significantly outperforming the previous state of the art (Smith'16), which achieved $\mathbf{7 2 . 4 6 \%}$. Furthermore, our new method is much simpler and more general than the previous approach. To demonstrate the generality of our model, we show that it also performs well on an even more challenging dataset derived from Google Street View, in which the goal is to extract business names from store fronts. Finally, we study the speed/accuracy tradeoff that results from using CNN feature extractors of different depths. Surprisingly, we find that deeper is not always better (in terms of accuracy, as well as speed). Our resulting model is simple, accurate and fast, allowing it to be used at scale on a variety of challenging real-world text extraction problems.
\end{abstract}

\section{INTRODUCTION}

Text recognition in an unconstrained natural environment is a challenging computer vision and machine learning problem. Traditional Optical Character Recognition (OCR) systems mainly focus on extracting text from scanned documents. Text acquired from natural scenes is more challenging due to visual artifacts, such as distortion, occlusions, directional blur, cluttered background or different viewpoints. Despite these difficulties, recent advances in deep learning have made significant progress on this problem [1]-[6].

In this paper, we concentrate not just on transcribing all the text in a given image, but instead on the harder problem of extracting a subset of useful pieces of information. The model has to focus on the important parts of the scene and to ignore visual clutter. We propose a model which leverages convolutional neural networks (CNNs), recurrent neural networks (RNNs), and a new form of spatial attention.

We benchmark our model on the French Street Name Signs dataset (FSNS) [7], derived from Google Street View. The dataset contains over 1M labeled images of visual text "in the wild"; this is significantly more than COCO Text [5], which only includes $63 \mathrm{k}$ labeled images. We achieve $84.2 \%$ accuracy on FSNS, significantly outperforming the previous state-ofthe-art [7], which achieved $72.46 \%$.

The previous state of the art method on FSNS [7] shifts different views of the same sign into the batch dimension, has multiple multilayer LSTMs designed to treat every line of text separately (up to 3 lines) and uses CTC loss. Our model is simpler, more accurate and makes fewer assumptions about the data. To demonstrate its broad applicability, we also evaluate our new model on the Street View Business Names dataset [8], showing strong results.

Finally, we study the accuracy and speed of using 3 different CNN-based feature extractors (namely inception-v2 [9], inception-v3 [10] and inception-resnet-v2 [10]) as input to our attention model. We find that inception-v3 and inceptionresnet-v2 perform comparably, and both significantly outperform inception-v2. Motivated by the need for speed, we also study the effect of using "ablated" versions of these models, which use fewer layers. Interestingly, we find that for all three networks, the accuracy initially increases with depth, but then starts to decrease. This is in contrast to models trained on the ILSVRC Imagenet dataset [11], which is comparable in size to FSNS. For image classification, accuracy tends to increase with depth monotonically. We believe the difference is that image classification needs very complicated features, which are spatially invariant, whereas, for text extraction, it hurts to use to use such features.

In summary, our contributions are as follows: (1) We present a novel attention-based text reading architecture, trained in an end-to-end way, that beats the previous state of the art on the FSNS dataset by a significant margin while being considerably simpler and more general. (2) We show how our new model also gives excellent results on a newer, even more challenging, Street View dataset. (3) We study the speed/accuracy tradeoff that results from using CNNs of different depths and recommend a configuration that is accurate and efficient.

The source code and a trained model are available at: https://github.com/tensorflow/models/tree/master/attention_ocr

\section{Methods}

In this section, we describe our model, which processes the image through a CNN, and then passes the (attentionally weighted) features into an RNN. See Figure 1 for a high level summary.

\section{A. CNN-based feature extraction}

We consider 3 kinds of CNN: inception-v2 [9], inception-v3 [10] and inception-resnet-v2 [10], which combines inception 


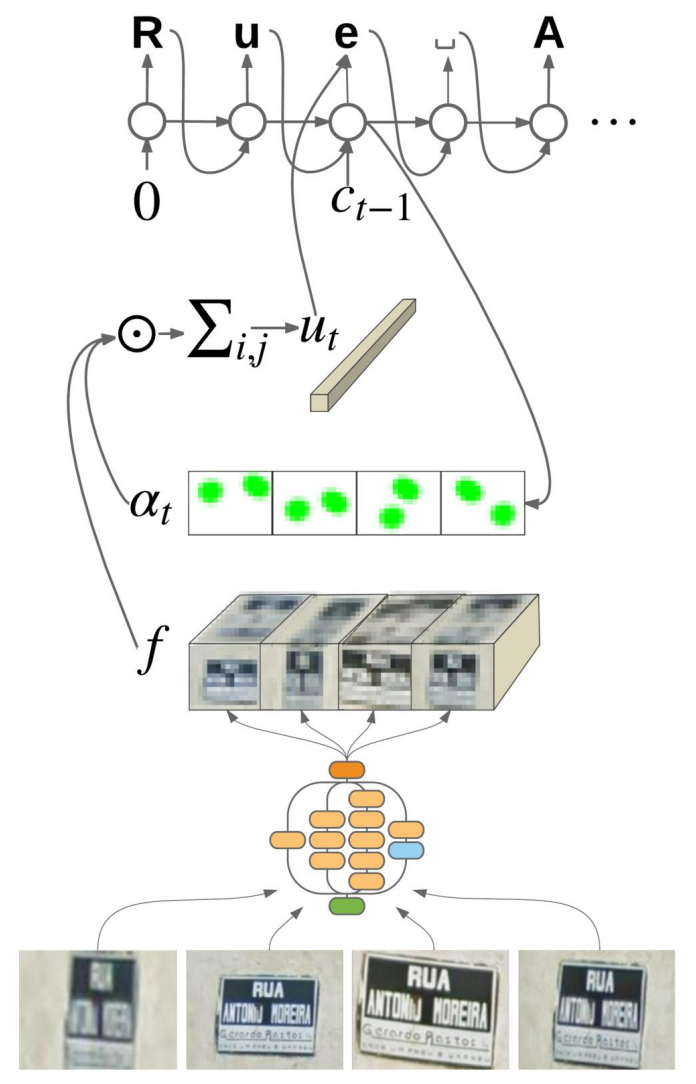

Fig. 1: Architecture of our model. We pass each of the four views through the same CNN feature extractor, and then concatenate the results into a single large feature map, shown by the cube labeled " $\mathrm{f}$ ". We take a spatially weighted combination to create a fixed-sized feature vector $u_{t}$, which is fed into the RNN.

with resnets [12]. These models achieve state-of-the-art performance on the Imagenet classification challenge [11], [13].

It has been shown in [14] that features from lower layers of CNNs trained on Imagenet transfer well to other tasks. However, this still leaves open the question of which layer to use as our feature representation. We study this in Section IV-B (We also compared the effects of pre-training on Imagenet vs. training from random initialization, and found no notable difference, so we only report results using the latter.)

We will use $f=\left\{f_{i, j, c}\right\}$ to denote the feature map derived by passing the image $x$ through a CNN (here $i, j$ index locations in the feature map, and $c$ indexes channels).

\section{B. $R N N$}

The main challenge is how to convert the feature maps into a single text string. Following previous work, we use an RNN (specifically, an LSTM [15]) for this; this acts as a characterlevel language model, which takes inputs from the image, as we explain below.

Let $s_{t}$ be the hidden state of the RNN at time $t$. The input to the RNN is determined by a spatially weighted combination of image features. This spatial attention mask is denoted by $\alpha_{t}=$ $\left\{\alpha_{t, i, j}\right\}$; we explain how to compute this in Section III-C Once we have computed the spatial mask, we compute a weighted combination of the features (the context) as follows:

$$
u_{t, c}=\sum_{i, j} \alpha_{t, i, j} f_{i, j, c}
$$

The total input to the RNN at time $t$ is defined as

$$
\hat{x}_{t}=W_{c} c_{t-1}^{\text {OneHot }}+W_{u_{1}} u_{t-1}
$$

where $c_{t-1}$ is the index of the previous letter (ground truth during training, predicted during test time). We then compute the output and next state of the RNN as follows:

$$
\left(o_{t}, s_{t}\right)=\operatorname{RNNstep}\left(\hat{x}_{t}, s_{t-1}\right)
$$

The final predicted distribution over letters at time $t$ is given by

$$
\hat{o}_{t}=\operatorname{softmax}\left(W_{o} o_{t}+W_{u_{2}} u_{t}\right)
$$

This combines information from the RNN, $o_{t}$, with information from the attentional feature vector, $u_{t}$. Finally, we compute the most likely letter:

$$
c_{t}=\arg \max _{c} \hat{o}_{t}(c)
$$

This is called greedy decoding.

\section{Spatial attention}

Most prior works that use spatial attention for OCR (e.g., [1], [16]-[20]) predict the mask based on the current RNN state, as follows:

$$
\begin{gathered}
a_{t, i, j}=V_{a}^{T} \tanh \left(W_{s} s_{t}+W_{f} f_{i, j,:}\right) \\
\alpha_{t}=\operatorname{softmax}_{i, j}\left(a_{t}\right)
\end{gathered}
$$

where $V_{a}$ is a vector and tanh is applied elementwise to its vector argument. This combines content from the image, via $W_{f} f$, with a time-varying offset, via $W_{s} s_{t}$, to determine where to look. We will use this as our baseline attention method.

The above attention mechanism is permutation invariant, meaning we could shuffle the order of the pixels and the mapping from $f$ to $\alpha_{t}$ would remain the same (since it is applied elementwise to each location). To make the model "location aware", we concatenate $f_{i, j}$ : with a one-hot encoding of the spatial coordinates $(i, j)$, as shown in Figure 2 More precisely, we replace the argument to the tanh function with the following:

$$
W_{s} s_{t}+W_{f_{1}} f_{i, j,:}+W_{f_{2}} e_{i}+W_{f_{3}} e_{j}
$$

where $e_{i}$ is a one-hot encoding of coordinate $i$, and similarly for $e_{j}$. This is equivalent to adding a spatially varying matrix of bias terms.

Our proposal is different than the location-aware attention mechanism proposed in [21]. They suggested adding $W_{a_{2}} * \alpha_{t-1}$ as input to the tanh function in Equation 6 where $W_{a_{2}}$ is a convolution kernel. However, in multiline text 


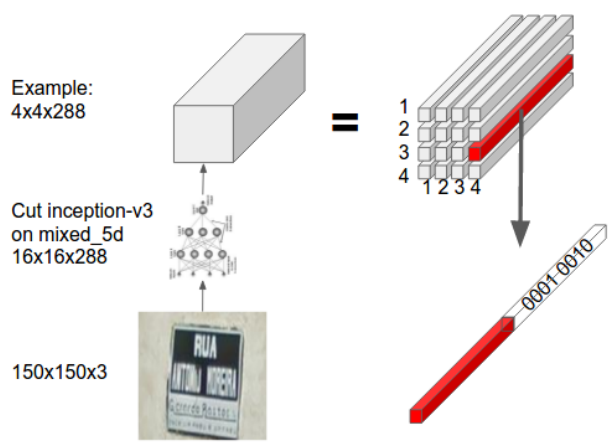

Fig. 2: Adding pixel coordinates to image features.

recognition problems, we sometimes have to make a big jump to the left side of the line below (see Figure 6 for example), which cannot be captured by this approach. (We tried their approach of location-aware attention, and it did not give good results for our problem, details omitted for brevity.)

\section{Handling multiple views}

In the FSNS dataset, we have four views for each input sign, each of size $150 x 150$. We process each of these independently, through the same $\mathrm{CNN}$-based feature extractor (parameters are shared), to compute four feature maps. We then concatenate these horizontally to create a single input feature map. For example, suppose the feature map for each of the four views is $16 \times 16 \times 320$; then after concatenation, the feature map $f_{i, j, c}$ will be $64 \times 16 \times 320$. (The actual spatial resolution of the feature maps varies, as we discuss in Section [V-B],

\section{E. Training}

We train the model using (penalized) maximum likelihood estimation, that is, we maximize $\sum_{t=1}^{T} \log p\left(y_{t} \mid y_{1: t-1}, x\right)$, where $x$ is the input image, $y_{t}$ is the predicted label for location $t$, and $T=37$ for the FSNS dataset. (If the output string is less than 37 characters, the model is required to predict a null character.) Since the model is autoregressive, we pass in the ground truth labels as history during training, as is standard [22]. Note that we do not need ground truth bounding boxes around any of the text, which makes collecting training data much easier.

Our training method allows us to use autoregressive connections easily, which is not possible when using CTC loss [23], which was used by the previous state of the art model on FSNS [7]. We find the use of such autoregressive dependencies improves accuracy by $6 \%$, and speeds up training $2 \mathrm{x}$ (details omitted for brevity).

We use stochastic gradient descent optimization with initial learning rate 0.002 , decay factor 0.1 after $1,200,000$ steps and momentum 0.75 . We finish training after 2,000, 000 steps. We use the following augmentation procedure per view: We randomly crop with the requirement to cover at least 0.8 area of the original input image and new aspect ratio to be between 0.8 and 1.2. After cropping we resize it to the initial size with one randomly chosen interpolation procedure: bilinear, bicubic, area, or nearest-neighbor. Then we apply random image distortions: change of contrast, hue, brightness, and saturation. To regularize the model, we use weight decay 0.00004, label smoothing 0.9 [9] and LSTM values clipping to 10. LSTM unit size is 256 . We use a batch size of 32 with asynchronous training on 40 machines. For inception-resnetv2 we have used a batch size of 12 due to GPU memory limitations. It takes less than 10 hours to train a single model with the inception-v3 network. We apply Polyak averaging [24] with decay 0.9999 to derive the weights for inference.

\section{DATASETS}

In this section, we describe the datasets that we use in our experiments.

\section{A. FSNS dataset}

The FSNS dataset [7] contains 965,917 training images, 38,633 validation images and 42, 839 test images. Each image has up to 4 tiles, intended to be a different view of the same physical street sign from Street View imagery from France. The size of every tile is $150 \times 150$ pixels. The first view represents the ground truth physical sign (as views do not always correspond to the same sign). See Figure 4 for some examples.

All the transcriptions of the street name are up to 37 characters long. (Our model takes advantage of this fact and always runs 37 steps, with an optional out-of-alphabet padded symbol.) There are 134 possible characters to choose from at each location, but most of the street names consist only of Latin letters.

\section{B. Street View Business Names Dataset}

This is an internal dataset which contains $\sim 1 M$ single view images of business storefronts extracted from Google Street View imagery. See Figure 5 for some examples. The size of every image is $352 \times 352$. All transcriptions contain up to 33 symbols, with 128 characters in the vocabulary.

This dataset is significantly more challenging than FSNS, as storefronts have a lot more variation, and richer contextual information, compared to the street name signs. Moreover, the business name can be a small fraction of the entire image.

\section{EXPERIMENTAL RESULTS}

In this section, we report our experimental results on various datasets.

\section{A. Accuracy on FSNS}

We use the full sequence accuracy metric to benchmark our model. It is a challenging metric, as it requires every character to agree with the ground truth. We compare the previous state-of-the-art [7] with five different versions of our model. In particular, we use three feature extractors (inception-v2, inception-v3 and inception-resnet-v2), and combine this with two attention models (standard and our novel position-dependent attention). As we see from Table $\Pi$ all our methods significantly outperform the previous state-of-the-art; inception-v 3 and inception-resnet-v2 give similar performance, 
TABLE I: Accuracy on FSNS test set.

\begin{tabular}{|c|c|c|}
\hline CNN & Attention & Accuracy \\
\hline Smith et al. [7] & NA & $72.46 \%$ \\
Inception-v2 & Standard & $80.7 \%$ \\
Inception-v2 & Location & $81.8 \%$ \\
Inception-v3 & Standard & $83.1 \%$ \\
Inception-v3 & Location & $84.0 \%$ \\
Inception-resnet-v2 & Standard & $83.3 \%$ \\
Inception-resnet-v2 & Location & $\mathbf{8 4 . 2} \%$ \\
\hline
\end{tabular}

and both significantly beat inception-v2; finally, we see that our novel location-aware attention helps by $0.9 \%$ over standard attention.

\section{B. The effect of depth on FSNS}

The main computational bottleneck in our model is the CNN-based feature extractor. To see how accuracy and speed vary as a function of the depth of the $\mathrm{CNN}$, we considered "cutting" standard models at different layers, and then using these as feature extractors. We evaluate the accuracy of the resulting trained model (measured as the percentage of full sequences predicted correctly), as well as the speed (measured in milliseconds per single image inference on Tesla K40 GPU). Table II shows the results for inception-v2, Table III shows the results for inception-v3, and Table IV] shows the results for inception-resnet-v2. Note that these results are using standard attention, not location-aware attention.

We see that the accuracy improves for a while, and then starts to drop as the depth increases. This trend holds for all three models. We believe the reason for this is that character recognition does not benefit from the high-level features that are needed for image classification. Also, the spatial resolution of the image features used as input for attention decreases after every max pooling operation, which limits the precision of the attention mask on a particular character. We don't see any dependency between accuracy and the theoretical receptive field of the neurons in the last convolutional layer, but the effective field of view can be much smaller.

When comparing the different architectures, we see that inception-resnet-v2 is the most accurate (0.833), then inception-v3 (0.831), and finally inception-v2 (0.807). We chose to use inception-v3 features from the mixed-5d layer for all the other experiments in this paper, since this is almost twice as fast as the best inception-resnet-v2 cut, and has very similar accuracy, it is optimal choice of the architecture for the given computational budget. For the fixed spatial resolution, processing time grows with the depth of the network. After the max pooling layer which decreases the spatial resolution of image features used in attention, we usually observe speed up in the processing time.

\section{Visualization of attention on FSNS}

To understand the behavior of our model, we use a visualization procedure similar to one proposed by [25]. For
TABLE II: Performance of different cuts of inception-v2. Mixed layers are cuts after the inception block of concatenated convolutions.

\begin{tabular}{|c|c|c|c|c|c|}
\hline $\begin{array}{c}\text { Inception-v2 } \\
\text { layer }\end{array}$ & $\begin{array}{c}\text { Size } \\
\text { per view }\end{array}$ & Acc & $\begin{array}{c}\text { ms/ } \\
\text { Image }\end{array}$ & Depth & $\begin{array}{c}\text { Rec. } \\
\text { Field }\end{array}$ \\
\hline MaxPool_3a_3x3 & $19 \times 19 \times 192$ & 0.539 & 26 & 3 & 27 \\
\hline Mixed_3b & $19 \times 19 \times 256$ & 0.777 & 31 & 6 & 59 \\
\hline Mixed_3c & $19 \times 19 \times 320$ & 0.803 & 37 & 9 & 91 \\
\hline Mixed_4a & $10 \times 10 \times 576$ & 0.765 & 34 & 12 & 155 \\
\hline Mixed_4b & $10 \times 10 \times 576$ & 0.789 & 37 & 15 & 219 \\
\hline Mixed_4c & $10 \times 10 \times 576$ & 0.805 & 38 & 18 & 283 \\
\hline Mixed_4d & $10 \times 10 \times 576$ & 0.804 & 41 & 21 & 347 \\
\hline Mixed_4e & $10 \times 10 \times 576$ & $\mathbf{0 . 8 0 7}$ & 44 & 24 & 411 \\
\hline Mixed_5a & $5 \times 5 \times 1024$ & 0.791 & 43 & 27 & 539 \\
\hline Mixed_5b & $5 \times 5 \times 1024$ & 0.760 & 45 & 30 & 667 \\
\hline Mixed_5c & $5 \times 5 \times 1024$ & 0.792 & 47 & 33 & 795 \\
\hline
\end{tabular}

TABLE III: Performance of different cuts of inception-v3.

\begin{tabular}{|c|c|c|c|c|c|}
\hline $\begin{array}{c}\text { Inception-v3 } \\
\text { layer }\end{array}$ & $\begin{array}{c}\text { Size } \\
\text { per view }\end{array}$ & Acc & $\begin{array}{c}\text { ms/ } \\
\text { Image }\end{array}$ & Depth & $\begin{array}{c}\text { Rec. } \\
\text { Field }\end{array}$ \\
\hline \hline MaxPool_3a_3x3 & $35 \times 35 \times 64$ & 0.157 & 26 & 3 & 15 \\
\hline Conv2d_3b_1x1 & $35 \times 35 \times 80$ & 0.541 & 31 & 4 & 15 \\
\hline Conv2d_4a_3x3 & $33 \times 33 \times 192$ & 0.674 & 37 & 5 & 23 \\
\hline MaxPool_5a_3x3 & $16 \times 16 \times 192$ & 0.712 & 35 & 5 & 31 \\
\hline Mixed_5b & $16 \times 16 \times 256$ & 0.818 & 29 & 8 & 63 \\
\hline Mixed_5c & $16 \times 16 \times 288$ & 0.816 & 33 & 11 & 95 \\
\hline Mixed_5d & $16 \times 16 \times 288$ & $\mathbf{0 . 8 3 1}$ & 36 & 14 & 127 \\
\hline Mixed_6a & $7 \times 7 \times 768$ & 0.822 & 34 & 17 & 159 \\
\hline Mixed_6b & $7 \times 7 \times 768$ & 0.804 & 37 & 22 & 351 \\
\hline Mixed_6c & $7 \times 7 \times 768$ & 0.822 & 40 & 27 & 543 \\
\hline Mixed_6d & $7 \times 7 \times 768$ & 0.820 & 42 & 32 & 735 \\
\hline Mixed_6e & $7 \times 7 \times 768$ & 0.826 & 45 & 37 & 927 \\
\hline Mixed_7a & $3 \times 3 \times 1280$ & 0.674 & 45 & 41 & 1033 \\
\hline Mixed_7b & $3 \times 3 \times 2048$ & 0.802 & 48 & 44 & 1161 \\
\hline Mixed_7c & $3 \times 3 \times 2048$ & 0.810 & 51 & 47 & 1289 \\
\hline
\end{tabular}

every predicted character $k$, we compute the partial derivative of its logit $a_{k}$ with respect to the input image $x$. We can then compute the saliency of pixel $(i, j)$ using the formula $v_{i, j}=\left\|\frac{\partial a_{k}}{\partial x_{i, j,:}}\right\|_{2}$, where we compute the L2 norm of the derivative across all the color channels. To get a less noisy saliency map, we create 16 slightly perturbed versions of the image (by adding a small amount of Gaussian noise) and then average the results. Additionally, we visualize the attention map $\alpha_{t}$ by upsampling with nearest-neighbor interpolation to the size of the input.

A visualization example is shown in Figure 3 . The red color represents the saliency map, and the green color represents the attention map. On the first time step, the attention map highlights the letter 'R,' which is the start of the name. Since 'Rue' is the most common beginning of a street name in this dataset, the model can predict the string 'Rue' without paying attention to the image for the next three iterations. This phenomenon is represented by the vertical green bars, which show that the model is just paying attention to the edge of the image, which has no informative content. We also see that the saliency map is spatially diffused in the first step since any of the letters ' $R$ ', ' $u$ ' or 'e' give evidence in support of 'Rue'; for the remaining three iterations, the saliency map is 
TABLE IV: Performance of different cuts of inception-resnetv2. We only report results from layer Mixed_5b and above as cuts for lower layers are identical to the inception-v3 network.

\begin{tabular}{|c|c|c|c|c|c|}
\hline $\begin{array}{c}\text { Inception-resnet-v2 } \\
\text { layer }\end{array}$ & $\begin{array}{c}\text { Size } \\
\text { per view }\end{array}$ & Acc & $\begin{array}{c}\text { ms/ } \\
\text { Image }\end{array}$ & Depth & $\begin{array}{c}\text { Rec. } \\
\text { Field }\end{array}$ \\
\hline Mixed_5b & $16 \times 16 \times 320$ & 0.818 & 43 & 8 & 63 \\
\hline Mixed_5b+5 & $16 \times 16 \times 320$ & 0.822 & 57 & 28 & 223 \\
\hline Mixed_5b+10 & $16 \times 16 \times 320$ & 0.824 & 66 & 48 & 383 \\
\hline Mixed_6a & $7 \times 7 \times 1088$ & $\mathbf{0 . 8 3 3}$ & 70 & 51 & 415 \\
\hline Mixed_6a+5 & $7 \times 7 \times 1088$ & 0.827 & 90 & 71 & 895 \\
\hline Mixed_6a +10 & $7 \times 7 \times 1088$ & 0.817 & 125 & 91 & 1375 \\
\hline Mixed_6a + 15 & $7 \times 7 \times 1088$ & 0.829 & 136 & 111 & 1855 \\
\hline Mixed_6a + 20 & $7 \times 7 \times 1088$ & 0.824 & 158 & 131 & 2335 \\
\hline Mixed_7a & $3 \times 3 \times 2080$ & 0.819 & 307 & 134 & 2399 \\
\hline Mixed_7a + 5 & $3 \times 3 \times 2080$ & 0.823 & 406 & 154 & 2719 \\
\hline Mixed_7a + 10 & $3 \times 3 \times 2080$ & 0.825 & 512 & 174 & 3039 \\
\hline Conv2d_7b_1x1 & $3 \times 3 \times 1536$ & 0.832 & 546 & 175 & 3039 \\
\hline
\end{tabular}

TABLE V: Breakdown of error types on FSNS.

\begin{tabular}{|c|c|}
\hline Error type & Percent \\
\hline \hline Wrong ground truth & 48 \\
\hline Wrong / Added / Missing accent over $e$ & 17 \\
\hline Wrong single letter inside the word & 9 \\
\hline $\begin{array}{c}\text { Wrong single letter at the } \\
\text { beginning / end of word }\end{array}$ & 8 \\
\hline Added / Missing hyphen (-) & 7 \\
\hline Wrong full word & 6 \\
\hline Read from the wrong view & 3 \\
\hline $\begin{array}{c}\text { Wrong / Added / Missing accent } \\
\text { over different letter than e }\end{array}$ & 2 \\
\hline
\end{tabular}

zero everywhere.

On the 9th step, the model correctly emits the letter 'F,' which is the start of the word 'Fonds'. Note how this word is blurred out in the first view (top left image): this kind of blurring occurs on some words in FSNS, due to mistakes in the license plate or face detection systems. Consequently, the attention and saliency maps are zero for the first view (left column) when processing 'Fonds'.

\section{Error Analysis on FSNS}

We analyze 100 randomly sampled wrong predictions to understand the weaknesses of our model better. $48 \%$ of the "errors" is due to the incorrect ground truth. Table $\mathrm{V}$ gives a more detailed breakdown. The most common error is due to the wrong accent over the letter e (it should be either é or è); interestingly, this is also the most common mistake in the ground truth transcription.

In Figure 4, we show some of the test cases where our model has a different prediction than the ground truth. (In the last example, this is due to an error in the ground truth.)

\section{E. Results on internal datasets}

We now report results on a new internal dataset. Since this dataset is not public, we just show qualitative results; the purpose is to show how the very same model can be used for extracting information from many different kinds of street signs.

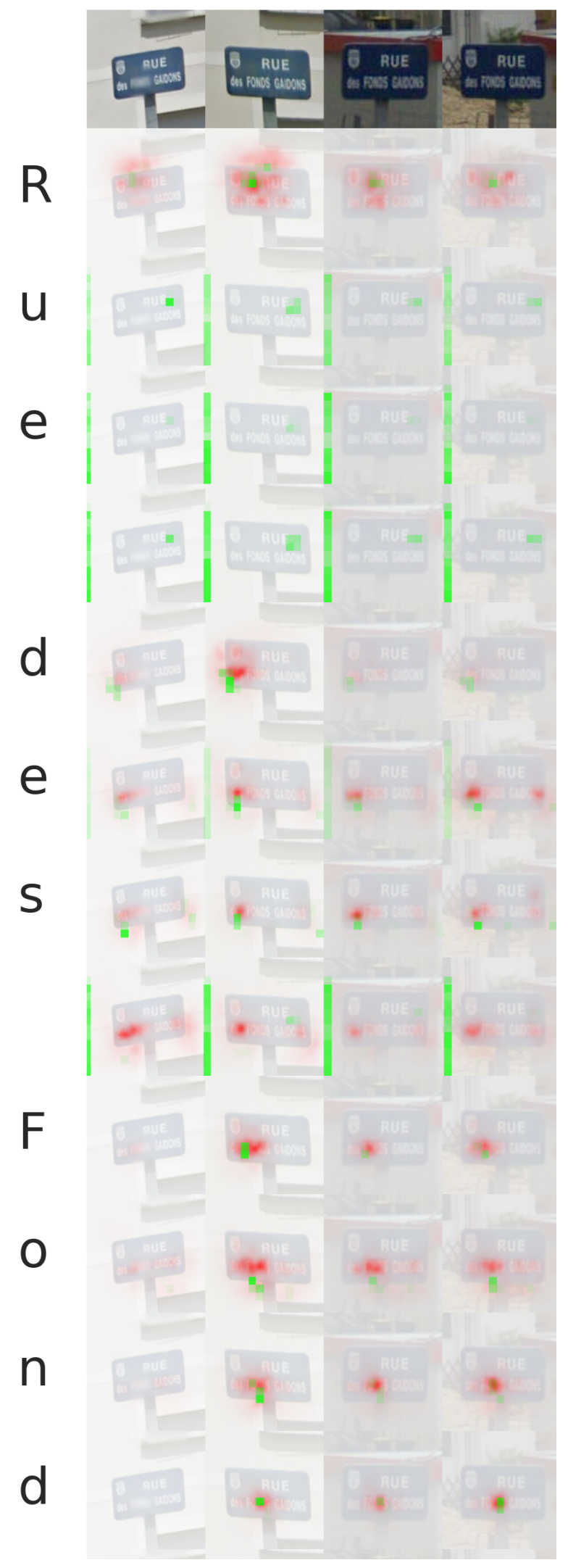

Fig. 3: Visualization of saliency maps (in red) and attention masks (in green) on an FSNS image. The model correctly predicts the string "Rue des Fonds Gadons"; we just show the first 12 steps. 

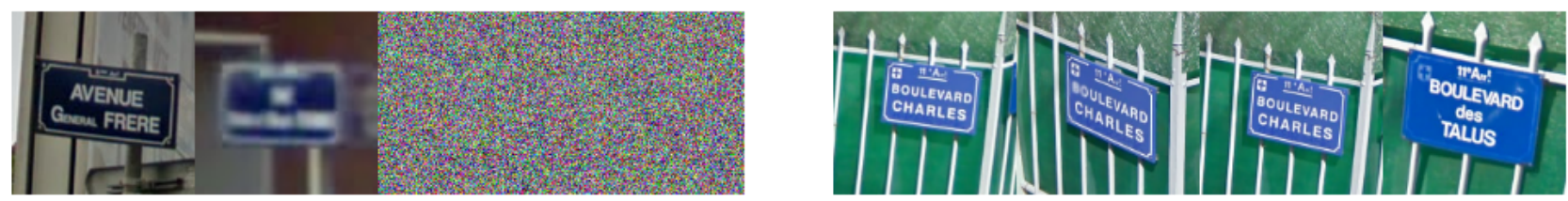

(a) Confused by font. Pred = 'Avenue Georges Frere', GT = 'Avenue General (b) Read text from the wrong view. Pred='Boulevard des Talus', Frere'. GT='Boulevard Charles'.
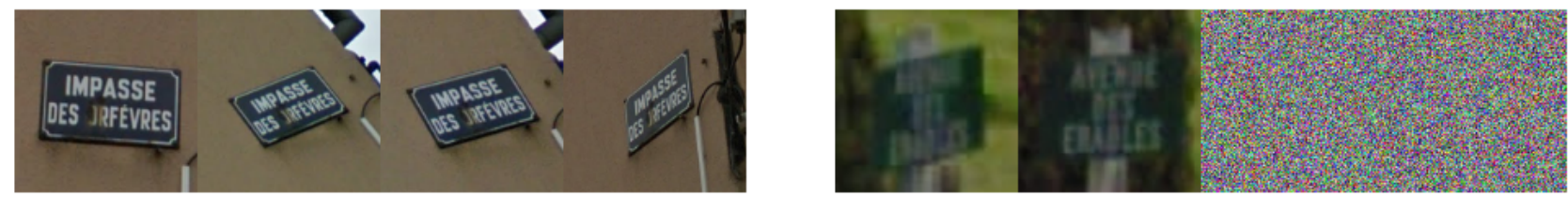

(c) Confusion due to scratched letter, which looks like ' $\mathrm{J}$ ', but model uses (d) The model has better language prior than the human annotator. its prior to produce 'O'. Pred='Impasse des Jorfèvres', GT='Impasse des Pred='Avenue des Erables', Wrong GT='Avenue des Enadles'. Orfévres'.

Fig. 4: Examples of FSNS signs where our prediction (Pred.) differs from ground truth (GT).
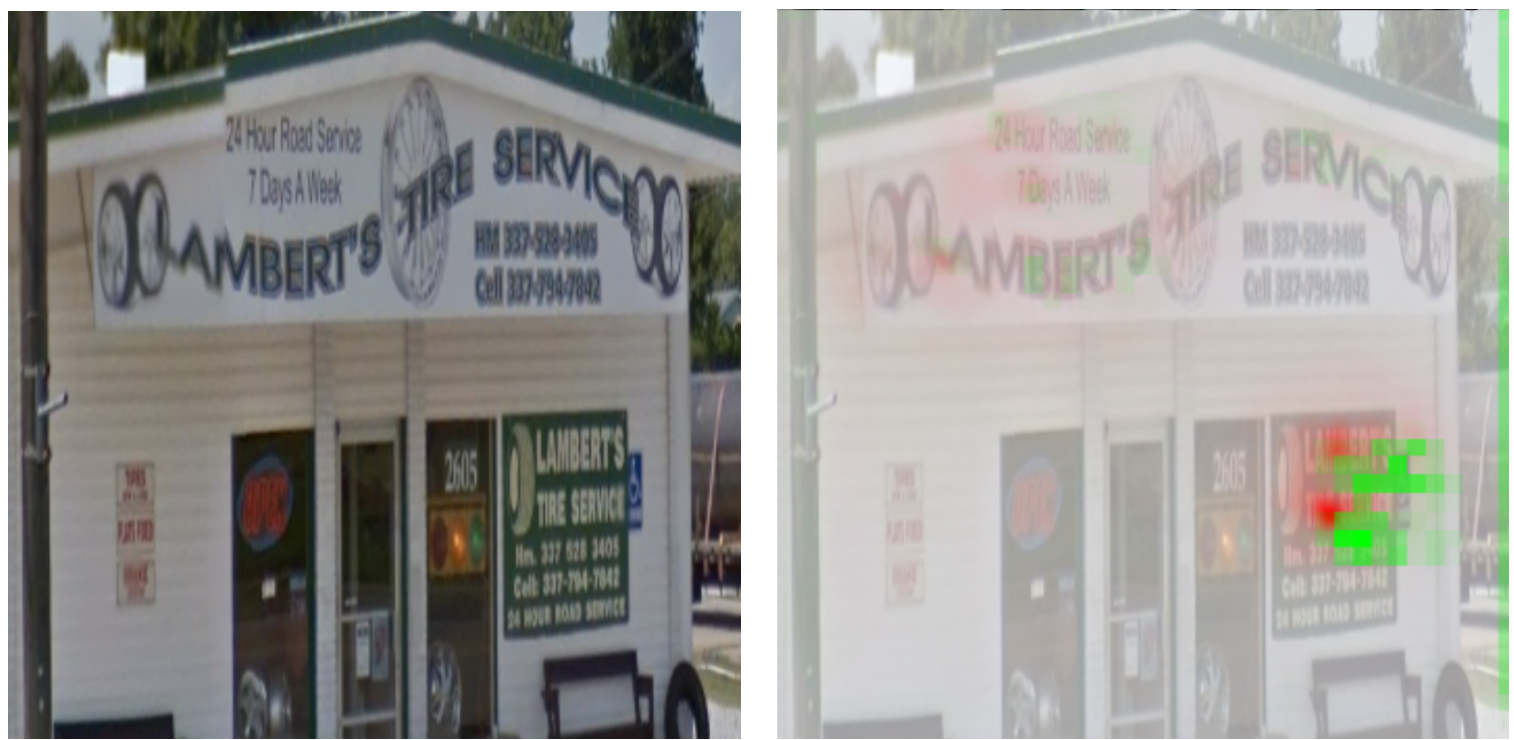

Fig. 5: Left: An example image from the internal Business Names dataset. The ground truth string is "Lambert's Tire Service", which is correctly predicted by the model. This name is written on the top of the store in a "wavy" font, but also on the right-hand side (green sign) in a more standard horizontal font. Right: Visualization of the time-averaged saliency maps (in red) and attention masks (in green). Notice how they focus on the location that contains the store name.

Figure 5 shows an example of our system applied to an image from the Business Names dataset. Notice how the name of the business, "Lambert's Tire Service", appears in two locations: at the top, and at the bottom right. The model chooses to attend to the latter, perhaps because the font is more standard, and the writing is horizontal and not "wavy".

Figure 6 shows another example. This time, we see that the attention maps scans left to right, to read "Autopartes", but has to jump down a line and all the way to the left (like the carriage return action of a mechanical typewriter) to read the second line of text, "Lubricantes Tauro".

\section{CONCLUSIONS AND FUTURE WORK}

We have presented an end-to-end approach for scene text recognition which gives state-of-the-art results on the challenging FSNS dataset, and an internal dataset. Our novel attention mechanism allows us to extract structured text information by reading only the interesting parts of the whole image. 


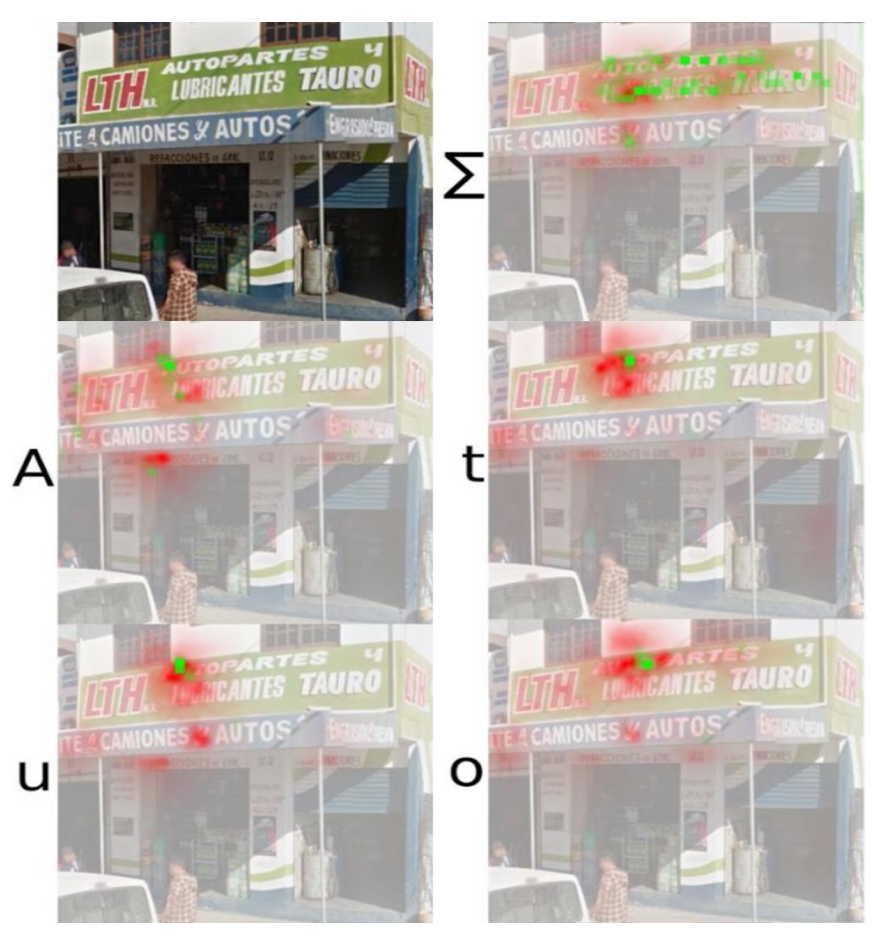

Fig. 6: Visualization of saliency maps (in red) and attention masks (in green) on a Business Names image. The model correctly predicts the string "Autopartes Lubricantes Tauro"; we just show the first few steps.

In the future, we would like to investigate more sophisticated ways of training the RNN, such as scheduled sampling [26] or hybrid ML/RL methods [27]. We would also like to extend the system to full structured extraction of business information from storefronts.

\section{ACKNOWLEDGMENT}

The authors would like to thank Sergio Guadarrama, Alex Lacoste, Ray Smith, Quoc Le, Christian Szegedy, Oriol Vinyals, Ilya Sutskever, Sergey Ioffe, Vincent Vanhoucke and Sacha Arnoud for valuable discussions and TensorFlow support.

\section{REFERENCES}

[1] T. He, W. Huang, Y. Qiao, and J. Yao, "Text-attentional convolutional neural network for scene text detection," IEEE Transactions on Image Processing, vol. 25, no. 6, pp. 2529-2541, 2016.

[2] K. Wang, B. Babenko, and S. Belongie, "End-to-end scene text recognition," in 2011 International Conference on Computer Vision. IEEE, 2011, pp. 1457-1464.

[3] M. Jaderberg, K. Simonyan, A. Vedaldi, and A. Zisserman, "Reading text in the wild with convolutional neural networks," International Journal of Computer Vision, vol. 116, no. 1, pp. 1-20, 2016.

[4] D. Karatzas, L. Gomez-Bigorda, A. Nicolaou, S. Ghosh, A. Bagdanov, M. Iwamura, J. Matas, L. Neumann, V. R. Chandrasekhar, S. Lu et al., "Icdar 2015 competition on robust reading," in Document Analysis and Recognition (ICDAR), 2015 13th International Conference on. IEEE, 2015, pp. 1156-1160
[5] A. Veit, T. Matera, L. Neumann, J. Matas, and S. Belongie, "Coco-text: Dataset and benchmark for text detection and recognition in natural images," arXiv preprint arXiv:1601.07140, 2016.

[6] M. Iwamura, T. Matsuda, N. Morimoto, H. Sato, Y. Ikeda, and K. Kise, "Downtown osaka scene text dataset," in European Conference on Computer Vision. Springer, 2016, pp. 440-455.

[7] R. Smith, C. Gu, D.-S. Lee, H. Hu, R. Unnikrishnan, J. Ibarz, S. Arnoud, and S. Lin, "End-to-end interpretation of the french street name signs dataset," in European Conference on Computer Vision. Springer, 2016 pp. 411-426.

[8] Q. Yu, C. Szegedy, M. C. Stumpe, L. Yatziv, V. Shet, J. Ibarz, and S. Arnoud, "Large scale business discovery from street level imagery," arXiv preprint arXiv:1512.05430, 2015.

[9] C. Szegedy, V. Vanhoucke, S. Ioffe, J. Shlens, and Z. Wojna, "Rethinking the inception architecture for computer vision," in Proceedings of the IEEE Conference on Computer Vision and Pattern Recognition, 2016, pp. 2818-2826.

[10] C. Szegedy, S. Ioffe, V. Vanhoucke, and A. A. Alemi, "Inception-v4, inception-resnet and the impact of residual connections on learning." in $A A A I, 2017$, pp. 4278-4284.

[11] O. Russakovsky, J. Deng, H. Su, J. Krause, S. Satheesh, S. Ma, Z. Huang, A. Karpathy, A. Khosla, M. Bernstein, A. C. Berg, and L. FeiFei, "Imagenet large scale visual recognition challenge," Intl. J. Comp. Vision, vol. 115, no. 3, pp. 211-252, 2015.

[12] K. He, X. Zhang, S. Ren, and J. Sun, "Deep residual learning for image recognition," in CVPR, 2016.

[13] A. Canziani, A. Paszke, and E. Culurciello, "An analysis of deep neural network models for practical applications," arXiv preprint arXiv:1605.07678, 2016.

[14] J. Yosinski, J. Clune, Y. Bengio, and H. Lipson, "How transferable are features in deep neural networks?" in Advances in neural information processing systems, 2014, pp. 3320-3328.

[15] S. Hochreiter and J. Schmidhuber, "Long short-term memory," Neural computation, vol. 9, no. 8, pp. 1735-1780, 1997.

[16] C.-Y. Lee and S. Osindero, "Recursive recurrent nets with attention modeling for ocr in the wild," in Proceedings of the IEEE Conference on Computer Vision and Pattern Recognition, 2016, pp. 2231-2239.

[17] B. Shi, X. Bai, and C. Yao, "An end-to-end trainable neural network for image-based sequence recognition and its application to scene text recognition," IEEE transactions on pattern analysis and machine intelligence, 2016.

[18] B. Shi, X. Wang, P. Lyu, C. Yao, and X. Bai, "Robust scene text recognition with automatic rectification," in Proceedings of the IEEE Conference on Computer Vision and Pattern Recognition, 2016, pp. $4168-4176$.

[19] T. Bluche, J. Louradour, and R. Messina, "Scan, attend and read: Endto-end handwritten paragraph recognition with mdlstm attention," arXiv preprint arXiv:1604.03286, 2016.

[20] T. Bluche, "Joint line segmentation and transcription for end-to-end handwritten paragraph recognition," arXiv preprint arXiv:1604.08352, 2016.

[21] J. K. Chorowski, D. Bahdanau, D. Serdyuk, K. Cho, and Y. Bengio, "Attention-based models for speech recognition," in Advances in Neural Information Processing Systems, 2015, pp. 577-585.

[22] I. Sutskever, O. Vinyals, and Q. V. Le, "Sequence to sequence learning with neural networks," in Advances in neural information processing systems, 2014, pp. 3104-3112.

[23] A. Graves, S. Fernández, F. Gomez, and J. Schmidhuber, "Connectionist temporal classification: labelling unsegmented sequence data with recurrent neural networks," in Proceedings of the 23rd international conference on Machine learning. ACM, 2006, pp. 369-376.

[24] B. T. Polyak and A. B. Juditsky, "Acceleration of stochastic approximation by averaging," SIAM Journal on Control and Optimization, vol. 30, no. 4, pp. 838-855, 1992.

[25] K. Simonyan, A. Vedaldi, and A. Zisserman, "Deep inside convolutional networks: Visualising image classification models and saliency maps," arXiv preprint arXiv:1312.6034, 2013.

[26] S. Bengio, O. Vinyals, N. Jaitly, and N. Shazeer, "Scheduled sampling for sequence prediction with recurrent neural networks," in Advances in Neural Information Processing Systems, 2015, pp. 1171-1179.

[27] M. Norouzi, S. Bengio, N. Jaitly, M. Schuster, Y. Wu, D. Schuurmans et al., "Reward augmented maximum likelihood for neural structured prediction," in Advances In Neural Information Processing Systems, 2016, pp. 1723-1731. 